\title{
Experimental estimation of mismatch uncertainty in radio - frequency power and attenuation measurements
}

\author{
Kamlesh Patel ${ }^{\star}$ \\ Department Of Electronic Science, University of Delhi South Campus, 110012 New Delhi, India
}

Received: 15 May 2015 / Accepted: 27 October 2015

\begin{abstract}
In this paper, the effects of the input quantity representations in linear and complex forms are analyzed to estimate mismatch uncertainty separately for one-port and two-port components. The mismatch uncertainties in power and attenuation measurements are evaluated for direct, ratio and substitution techniques with the use of a vector network analyzer system in the range of 1 to $18 \mathrm{GHz}$. The estimated mismatch uncertainties were compared for the same device under test and these values have verified that their evaluation is dependent on the representations of input quantities. In power measurements, the mismatch uncertainty is reduced when evaluating from the voltage standing wave ratio or reflection coefficient magnitudes in comparison to the complex reflection coefficients. The mismatch uncertainty in the attenuation measurements, are found higher and linearly increasing while estimating from the linear magnitude values than those from the $S$-parameters of the attenuator. Thus in practice, the mismatch uncertainty is estimated more accurately using the quantities measured in the same representations as of measuring quantity.
\end{abstract}

Keywords: Mismatch uncertainty estimation, complex quantity, RF impedance, vector network analyzer measurement

\section{Introduction}

One of the most important and dominant factors in the microwave transmission measurements is the impedance mismatch, lot of efforts are made to minimize and ensure the maximum power transmission through the microwave system. The main cause of the mismatch is the deviation in the connecting port dimensions from the standard dimensions and characteristics of the device under test (DUT) either in coaxial or waveguide configurations [1-4]. However, the connector size and flange dimension are fairly standardized now and the mismatch is caused by the component itself after the connector. Every calibration is associated with an uncertainty value and uncertainties due to mismatch become an important contributor in the overall uncertainty analysis especially above $30 \mathrm{MHz}$. The evaluation of mismatch uncertainty plays a vital role in precision radio frequency attenuation and power measurements. The mismatch uncertainties of complex quantities are studied for various cases with examples with any phase information $[5,6]$. The available mismatch uncertainty formulas have raised another issue of how and where to use a particular formula for a new user either in RF calibration or in measurement. So, the user is spending more time in searching and implementing the best formula depending on the parameter being measured,

${ }^{\star}$ Correspondence: kpatel@south.du.ac.in input parameters, measurement setup, type of representations, complex or linear etc. An effort was made to address this issue for RF engineers, the formulas have been presented with the sufficient references to give a glimpse of their appropriate and suitable applications at a single place [7]. However, further work was required in this area, first to study the response of the equations available in different linear and complex representations of input quantities. The second is to check whether the estimated uncertainty values are obtained same or differently for the given DUT at the particular frequency using any formula. And the third, to identify the reason, if there are any changes due to other factors except the complex representations of the input quantities of the power and attenuation measurement ranges, independently.

So, the present paper deals to find solutions of these issues and explored the effect of input representations used to experimentally estimate the mismatch uncertainty in accordance with the standard documents [8-10]. We have performed the measurements to apply on various mismatch uncertainty formulas in terms of magnitude only (linear or $\mathrm{dB}$ ), magnitude/phase components ( $\mathrm{dB}$ and linear) and real/imaginary components. For example, power can be measured in terms of watts or $\mathrm{dBm}$ at the same time depending on the purpose or DUT, whereas attenuation is measured in terms of the unitless (linear) quantity or dB. So, it is very important to study the dependence of input quantity representation, DUT parameter 
and its unit on the overall evaluation of mismatch uncertainty. In this paper, appropriate calculated results based on the measurements are presented for various cases of power and attenuation calibrations. The responses of various uncertainty expressions are analyzed through the measurements have been performed for different DUTs, thermistor mount, fixed attenuator and step attenuator in 1-18 GHz range using vector network analyzer (VNA) Wiltron 37247B by applying short-open-load-thru (SOLT) calibration [11]. The various reflection coefficient values of load $\left(\Gamma_{\mathrm{L}}\right)$, DUT $\left(\Gamma_{\mathrm{DUT}}\right)$, impedance standard $\left(\Gamma_{\mathrm{Std}}\right)$ etc. are measured. The $S$-parameters of these DUTs are also measured in complex and linear formats. The associated uncertainties of these measured parameters are evaluated independently as per GUM documents $[9,10]$. In the last section, the practical results from the methods to control mismatch uncertainty are discussed.

\section{Estimation of mismatch uncertainty in power}

\subsection{Mismatch uncertainty for a simple power measurement}

In this case, mismatch uncertainty for power in $\mathrm{dB}$ can be expressed as,

$$
M P(\mathrm{~dB})=8.686\left|\Gamma_{\mathrm{G}}\right|\left|\Gamma_{\mathrm{DUT}}\right|
$$

where, $\Gamma_{\mathrm{G}}$ is the linear reflection coefficient magnitude of source and $\Gamma_{\mathrm{DUT}}$ is the linear reflection coefficient magnitude of device under test (DUT).

Using the source and DUT reflection coefficients $\left(\Gamma_{\mathrm{G}}\right.$ and $\left.\Gamma_{\mathrm{DUT}}\right)$ in terms of magnitude and phase, it will be given as:

$$
M P_{m p}=8.686\left|\Gamma_{\mathrm{G}}\right|\left|\Gamma_{\mathrm{DUT}}\right| \cos \theta_{\mathrm{DUT}}
$$

where $\theta_{\text {DUT }}$ is the relative phase of $\Gamma_{\mathrm{G}}$ and $\Gamma_{\mathrm{DUT}}$. If only source's VSWR is available, it will be the phase associated with $\Gamma_{\text {DUT }}$ in equation (2).

In terms of the real and imaginary components, if $\Gamma_{\text {DUT }}=p+j q$, it can be written as,

$$
M P_{r i}=8.686\left(\left|\Gamma_{\mathrm{G}}\right| \cdot \sqrt{p^{2}+q^{2}}\right) .
$$

A thermistor mount model no. M1110 is taken as a DUT and its reflection coefficient values $\left(\Gamma_{\text {DUT }}\right)$ are measured using VNA. These measured values along with their associated uncertainties from VNA and source reflection coefficient $\left(\Gamma_{\mathrm{G}}\right)$ of 0.231 (VSWR $\approx 1.6$ ) have been applied in equation (1) to estimate the mismatch uncertainties [7]. The mismatch uncertainty calculated using equations (2) and (3) for the thermistor mount are shown in Figure 1a. These mismatch uncertainties are found slightly increasing with the frequency in average range of less than $0.05 \mathrm{~dB}$. The uncertainties calculated by complex values are more spread and higher ( 0.01 to $0.13 \mathrm{~dB}$ ) than the uncertainties obtained from the reflection coefficient magnitudes only in

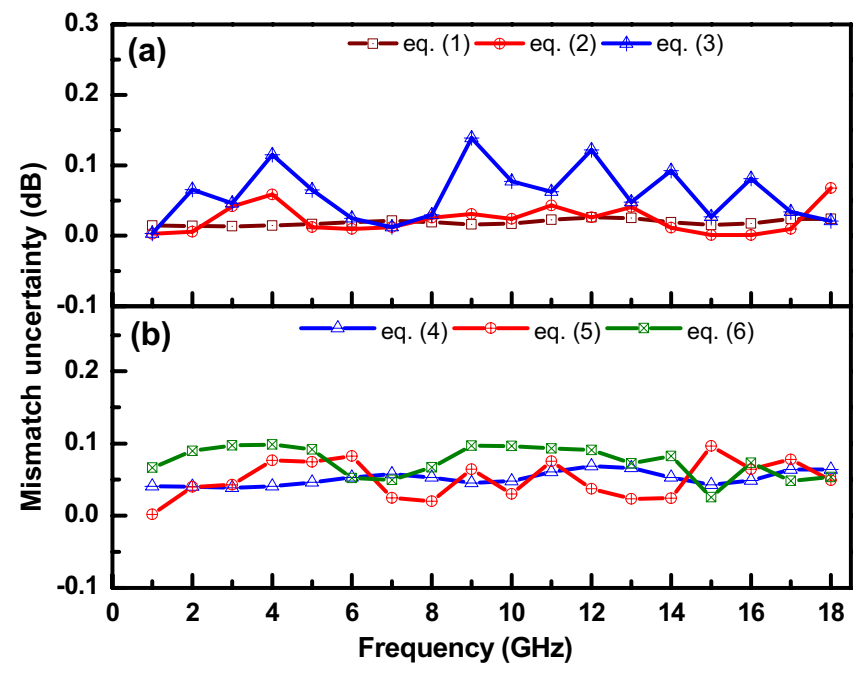

Fig. 1. Mismatch uncertainty for $0.0 \mathrm{dBm}$ power in (a) direct power measurement, and (b) comparison power measurement.

the same range of frequencies. However, it is expected that for the power measurements other than $0.0 \mathrm{~dB}$, the uncertainty values will differ according to the VSWR (or $\Gamma$ ) variation of thermistor mount.

\subsection{Mismatch uncertainty for a comparison power measurement}

The expression for the mismatch uncertainty for null comparison power measurement $M N P_{\Gamma}$ has modified at $50 \mathrm{MHz}\left(f_{1}\right)$ and the measurement frequency $\left(f_{2}\right)$ and given as [7],

$$
\begin{aligned}
M N P_{\Gamma}= & 8.686\left(\left\langle\left|\Gamma_{\mathrm{G}}\right|\left(\left|\Gamma_{\mathrm{DUT}}\right|+\left|\Gamma_{\mathrm{Std}}\right|\right)\right\rangle_{f_{1}}\right. \\
& \left.+\left\langle\left|\Gamma_{\mathrm{G}}\right|\left(\left|\Gamma_{\mathrm{DUT}}\right|+\left|\Gamma_{\mathrm{Std}}\right|\right)\right\rangle_{f_{2}}\right) .
\end{aligned}
$$

In terms of complex values, the following formulas are useful,

$$
\begin{aligned}
& M N P_{m p}=8.686 \\
& \times\left(\begin{array}{c}
\left\langle\left|\Gamma_{\mathrm{G}}\right|\left(\left|\Gamma_{\mathrm{DUT}}\right| \cos \theta_{\mathrm{DUT}}+\left|\Gamma_{\mathrm{Std}}\right| \cos \theta_{\mathrm{Std}}\right)\right\rangle_{f_{1}} \\
+\left\langle\left|\Gamma_{\mathrm{G}}\right|\left(\left|\Gamma_{\mathrm{DUT}}\right| \cos \theta_{\mathrm{DUT}}+\left|\Gamma_{\mathrm{Std}}\right| \cos \theta_{\mathrm{Std}}\right)\right\rangle_{f_{2}}
\end{array}\right)
\end{aligned}
$$

where, $\Gamma_{\mathrm{Std}}$ is the reflection coefficient of impedance standard (pre-calibrated) and $\theta_{\text {Std }}$ is the phase associated with $\Gamma_{\text {Std }}$.

In terms of the real and imaginary components, the expression for the mismatch uncertainty for null comparison power measurement $M N P_{r i}$ is given by:

$$
\begin{aligned}
M N P_{r i}= & 8.686\left(\left\{\left|\Gamma_{\mathrm{G}}\right|\left(\sqrt{p^{2}+q^{2}}+\sqrt{r^{2}+s^{2}}\right)\right\}_{f_{1}}\right. \\
& \left.+\left\{\left|\Gamma_{\mathrm{G}}\right|\left(\sqrt{p^{2}+q^{2}}+\sqrt{r^{2}+s^{2}}\right)\right\}_{f_{2}}\right)
\end{aligned}
$$

where $\Gamma_{\mathrm{DUT}}=p+j q$ and $\Gamma_{\mathrm{Std}}=r+j s$. 
A source $V S W R \approx 1.23$ is taken from the manufacturer specifications and respective measured values of $\mathrm{RF}$ load are fed in equations (4)-(6). As presented in Figure $1 \mathrm{~b}$, these evaluated uncertainties using the complex values are less than $0.1 \mathrm{~dB}$ and have a non-linear behavior. At higher frequencies, the reduced mismatch uncertainties remain constant with the frequency. However, the phase values control the mismatch behavior in equations (5) and (6) and so, it caused the randomly orientated higher uncertainties with frequency. For traceable complex reflection coefficient measurements, one may have higher values and random nature of mismatch uncertainties while estimating from the complex values.

\section{Estimation of mismatch uncertainty in attenuation}

\subsection{Mismatch uncertainty for a fixed attenuator}

For a fixed attenuator $(<10 \mathrm{~dB})$, when only magnitude is known (linear), total uncertainty contribution $M A_{T}$ due to mismatch error is given by [7]:

$$
\begin{aligned}
& M A_{T}(\mathrm{~dB})=20 \log _{10} \\
& \times \frac{1 \pm\left(\left|\Gamma_{\mathrm{G}} S_{11}\right|+\left|\Gamma_{\mathrm{L}} S_{22}\right|+\left|\Gamma_{\mathrm{G}} \Gamma_{\mathrm{L}} S_{11} S_{22}\right|+\left|\Gamma_{\mathrm{G}} \Gamma_{\mathrm{L}} S_{21} S_{12}\right|\right)}{1 \mp\left|\Gamma_{\mathrm{G}}\right|\left|\Gamma_{\mathrm{L}}\right|},
\end{aligned}
$$

where $S_{11}, S_{22}$ and $S_{21}, S_{12}$ are the reflection and transmission scattering coefficients of the attenuator.

If an attenuator has very small reflections at the ports and the source and load connected to it, are properly matched, the values of scattering parameters $\left(S_{11}\right.$ and $\left.S_{22}\right)$ and the reflection coefficients are extremely small, $\Gamma_{\mathrm{G}}$ and $\Gamma_{\mathrm{L}} \ll 1$. The maximum mismatch uncertainty in $\mathrm{dB}$ for attenuation is given by [7],

$$
\begin{aligned}
& M A_{S}(\mathrm{~dB})=8.686 \\
& \quad \times\left[\left|\Gamma_{\mathrm{G}}\right|\left|S_{11}\right|+\left|\Gamma_{\mathrm{L}}\right|\left|S_{22}\right|+\left|\Gamma_{\mathrm{G}} \Gamma_{\mathrm{L}}\right|\left(1+\left|S_{21} S_{12}\right|\right)\right] .
\end{aligned}
$$

In Intermediate frequency (IF) substitution technique for attenuation measurement, the mismatch uncertainty in terms of VSWR values is expressed by [7],

$$
\begin{aligned}
M A_{\text {vswr }}(\mathrm{dB})= & \pm 2\left(\left(V S W R_{\mathrm{G}}-1\right)\left(V S W R_{\mathrm{DUTI}}-1\right)\right. \\
& \left.+\left(V S W R_{\mathrm{L}}-1\right)\left(V S W R_{\mathrm{DUTO}}-1\right)\right)
\end{aligned}
$$

where $V S W R_{\mathrm{G}}$ is the measured $V S W R$ at the generator port, $V S W R_{\mathrm{L}}$ is the measured $V S W R$ at the load or detector port, $V S W R_{\text {DUTI }}$ is measured $V S W R$ at the input port of the attenuator (DUT), and $V S W R_{\text {DUTO }}$ is measured $V S W R$ at the output port of the attenuator (DUT).

In a second type of attenuation measurement, where the complex $S$-parameters are measured, the mismatch uncertainty is estimated differently. For such measurement

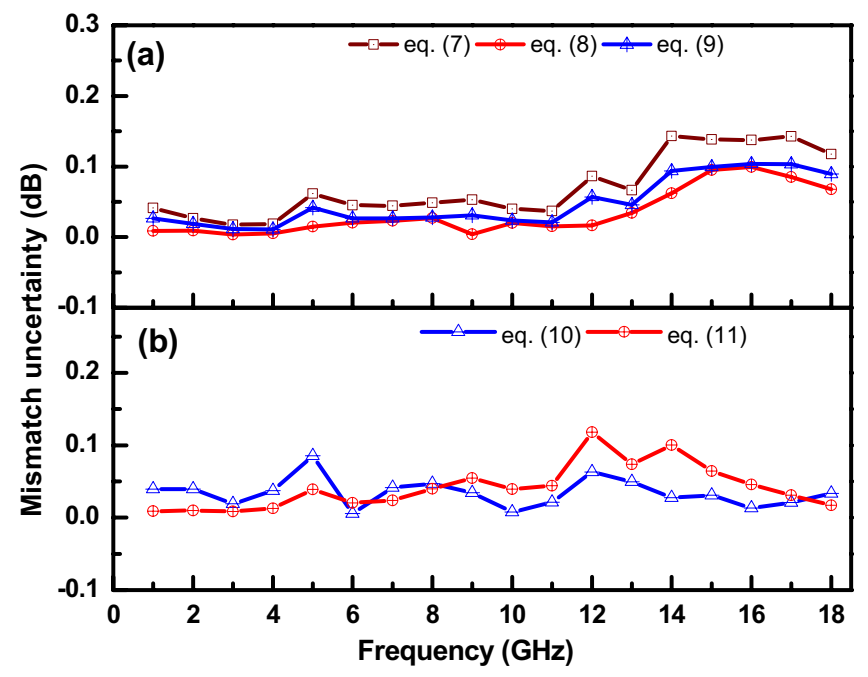

Fig. 2. Mismatch uncertainty for fixed attenuation measurement (a) using linear numbers, and (b) using complex numbers.

facility, the mismatch uncertainty can be evaluated in $\mathrm{dB}$ for high attenuation values $(>10 \mathrm{~dB})$ and given by [7].

$$
\begin{aligned}
& M A_{m p}(\mathrm{~dB})=8.686 \\
& \times\left(\begin{array}{l}
\left|\Gamma_{\mathrm{G}}\right|\left|\Gamma_{\mathrm{L}}\right|\left\langle\cos \left(\varphi_{\Gamma_{\mathrm{L}}}\right)-\left|S_{21}\right|\left|S_{12}\right| \cos \left(\varphi_{\Gamma_{\mathrm{L}}}+\varphi_{12}+\varphi_{21}\right)\right\rangle \\
-\left|\Gamma_{\mathrm{G}}\right|\left|S_{11}\right| \cos \left(\varphi_{11}\right)-\left|\Gamma_{\mathrm{L}}\right|\left|S_{22}\right| \cos \left(\varphi_{\Gamma_{\mathrm{L}}}+\varphi_{22}\right)
\end{array}\right)
\end{aligned}
$$

where $\varphi_{\Gamma}$ are the phase values associated with the respective reflection coefficients $(\Gamma)$ and $\phi_{n n}$ are the phase values associated with $S$-parameters as per the subscripts $(n=1,2)$.

When the $S$-parameters are measured as real and imaginary components, the mismatch uncertainty is [7],

$$
\begin{aligned}
M A_{r i}(\mathrm{~dB})= & 8.686\left(\left|\Gamma_{\mathrm{G}}\right| \sqrt{a^{2}+b^{2}}+\left|\Gamma_{L}\right| \sqrt{e^{2}+f^{2}}\right. \\
& \left.+\left|\Gamma_{\mathrm{G}}\right|\left|\Gamma_{L}\right|\left(\sqrt{(p k-q l)^{2}+(p l-q k)^{2}}\right)\right)
\end{aligned}
$$

where, $S_{11}=a+j b, S_{22}=e+j f, S_{21}=k+j l, S_{12}=p+j q$.

The VSWR values and linear $S$-parameters of a $50 \mathrm{~dB}$ attenuator (maximum $V S W R \approx 1.05$ ) are measured in a better matched measurement system. The evaluated mismatch uncertainties are obtained from equations (7)-(9) and have shown in Figure 2a. In such cases, the values of mismatch uncertainty are increasing from 0.01 to $0.15 \mathrm{~dB}$ and showing almost the similar trends with the frequency, irrespective of the input reflection coefficient values. However, a contribution of the measured attenuation is the reason behind a nominal difference between the responses of equations (8) and (9) and values are found within $0.12 \mathrm{~dB}$.

The mismatch uncertainties obtained from equations (10) and (11) are presented in Figure 2b. These responses estimated to 0.01 to 0.1 in $\mathrm{dB}$ and have not shown any similar values even at a single frequency. This means, these uncertainties are independent, while evaluating for the same DUT and are decreasing at the higher 


$$
M S A_{r i}(\mathrm{~dB})=8.686\left(\begin{array}{l}
\left|\Gamma_{\mathrm{G}}\right| \sqrt{(a-c)^{2}+(b-d)^{2}}+\left|\Gamma_{\mathrm{L}}\right| \sqrt{(e-g)^{2}+(f-h)^{2}} \\
+\left|\Gamma_{\mathrm{G}}\right|\left|\Gamma_{\mathrm{L}}\right| \sqrt{(p k-q l-m r+n s)^{2}+(p l+q k-m s-n r)^{2}}
\end{array}\right)
$$

frequencies. This supports a conclusion that the individual uncertainty contribution associated with each measured parameter influenced the final values of mismatch uncertainties and their estimation.

\subsection{Mismatch uncertainty for a step attenuator}

In this case, linear $S$-parameters are measured at every attenuation steps and along with the source and load reflection coefficient values, the maximum mismatch uncertainty is estimated using equation (12) in a matched system [7].

$$
\begin{gathered}
M S A(\mathrm{~dB})=8.686 \\
\times\left(\begin{array}{l}
\left|\Gamma_{\mathrm{G}}\right|^{2}\left(\left|S_{11}^{\prime}\right|^{2}-\left|S_{11}\right|^{2}\right)+\left|\Gamma_{\mathrm{L}}\right|^{2}\left(\left|S_{22}^{\prime}\right|^{2}-\left|S_{22}\right|^{2}\right) \\
+\left|\Gamma_{\mathrm{G}}\right|^{2}\left|\Gamma_{\mathrm{L}}\right|^{2}\left(\left|S_{21}^{\prime}\right|^{2}\left|S_{12}^{\prime}\right|^{2}-\left|S_{21}\right|^{2}\left|S_{12}\right|^{2}\right)
\end{array}\right)^{1 / 2},
\end{gathered}
$$

where $\Gamma_{\mathrm{G}}$ is the effective source port match, $\Gamma_{\mathrm{L}}$ is the effective load match, $S_{11}, S_{12}, S_{21}, S_{22}$ are the $S$-parameters of the attenuator (at the measuring attenuation level) and $S_{11}^{\prime}, S_{12}^{\prime}, S_{21}^{\prime}$, and $S_{22}^{\prime}$ are the $S$-parameters of the attenuator at the initial state ' 0 ' $\mathrm{dB}$.

Mismatch uncertainty using the input and output reflection coefficients of step attenuator in $\mathrm{dB}$, is given by equation (13) [7],

$$
\begin{aligned}
M S A_{\Gamma}(\mathrm{dB})= & 20 \log _{10}\left(1-\Gamma_{\mathrm{G}}\left(\Gamma_{\mathrm{i} 1}-\Gamma_{\mathrm{i} 2}\right)-\Gamma_{\mathrm{L}}\left(\Gamma_{\mathrm{o} 1}-\Gamma_{\mathrm{o} 2}\right)\right. \\
& \left.+\Gamma_{\mathrm{G}} \Gamma_{\mathrm{L}}\left(10^{-A_{1} / 10}-10^{-A_{2} / 10}\right)\right)
\end{aligned}
$$

where, $\Gamma_{\mathrm{L}}$ is a load reflection coefficient at the detector port, $\Gamma_{\mathrm{G}}$ is a generator (source) reflection coefficient at the generator port, $\Gamma_{\mathrm{i} 1}$ and $\Gamma_{\mathrm{i} 2}$ are the measured reflection coefficients of the step attenuator at ' 0 ' $\mathrm{dB}$ and measuring attenuation level settings of attenuator at the input port, $\Gamma_{\mathrm{o} 1}$ and $\Gamma_{\mathrm{o} 2}$ are the measured reflection coefficients of the step attenuator at ' 0 ' $\mathrm{dB}$ and measuring attenuation level settings of attenuator at the input port. $A_{1}$ ('0' $\mathrm{dB}$ ) and $A_{2}$ are the measured attenuation values of the step attenuator for two different settings of attenuator.

For high attenuation values, the mismatch uncertainty in $\mathrm{dB}$ can expressed in terms of complex $S$-parameters of attenuator in magnitude and phase and is given by [7],

$$
\begin{aligned}
& M S A_{m p}(\mathrm{~dB})=8.686 \\
& \times\left(\begin{array}{l}
\left|\Gamma_{\mathrm{G}}\right|\left\langle\left|S_{11}^{\prime}\right| \cos \left(\varphi_{11}^{\prime}\right)-\left|S_{11}\right| \cos \left(\varphi_{11}\right)\right\rangle \\
+\left|\Gamma_{\mathrm{G}}\right|\left|\Gamma_{\mathrm{L}}\right|\left\langle\left|S_{21}^{\prime}\right|\left|S_{12}^{\prime}\right| \cos \left(\varphi_{\Gamma_{\mathrm{L}}}+\varphi_{12}^{\prime}+\varphi_{21}^{\prime}\right)\right. \\
\left.-\left|S_{21}\right|\left|S_{12}\right| \cos \left(\varphi_{\Gamma_{\mathrm{L}}}+\varphi_{12}+\varphi_{21}\right)\right\rangle \\
+\left|\Gamma_{\mathrm{L}}\right|\left\langle\left|S_{22}^{\prime}\right| \cos \left(\varphi_{\Gamma_{\mathrm{L}}}+\varphi_{22}^{\prime}\right)-\left|S_{22}\right| \cos \left(\varphi_{\Gamma_{\mathrm{L}}}+\varphi_{22}\right)\right\rangle
\end{array}\right),
\end{aligned}
$$

where all $\phi$ values are defined as for equation (10).

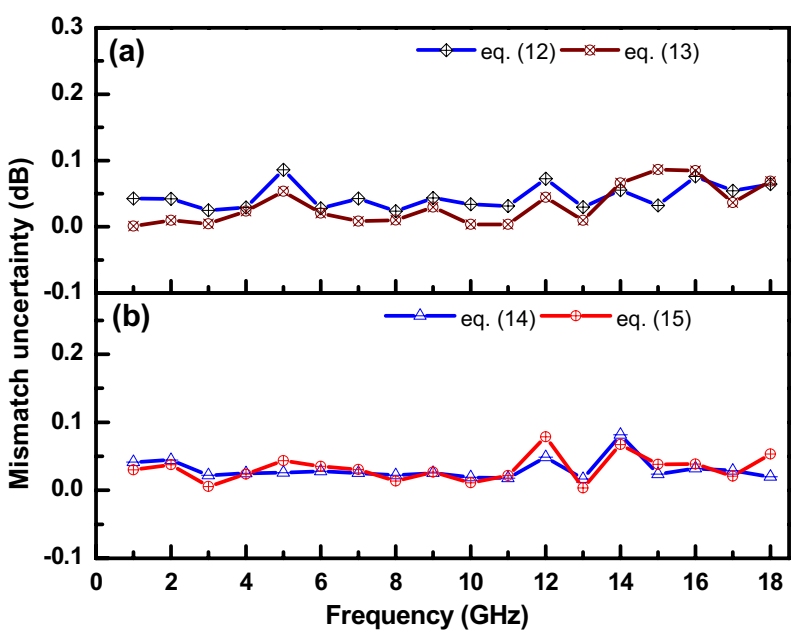

Fig. 3. Mismatch uncertainty for variable attenuation measurement (a) using the linear numbers, and (b) using the complex numbers.

In terms of real and imaginary representation for the measured complex $S$-parameters, $S_{11}=a+j b, S_{11}^{\prime}=c+$ $j d, S_{22}=e+j f, S_{22}^{\prime}=g+j h, S_{21}=k+j l, S_{21}^{\prime}=m+j n$, $S_{12}=p+j q, S_{12}^{\prime}=r+j s . \Gamma_{\mathrm{G}}$ and $\Gamma_{\mathrm{L}}$ are an effective test port match and effective load match respectively for $S_{12}$.

So, the mismatch uncertainty is then expressed as [7],

$$
\text { see equation (15) above. }
$$

An Agilent step attenuator at $0 \mathrm{~dB}$ and $10 \mathrm{~dB}$ setting was measured to extract its $S$-parameters values in complex and linear representations. The values of $\Gamma_{\mathrm{L}}$ and $\Gamma_{\mathrm{G}}$ were measured better than $30 \mathrm{~dB}$ at the insertion ports in the frequency range under consideration. The mismatch uncertainties are found in the range of 0.01-0.09 dB using equations (12) and (13) and shown in Figure 3a. The differences between these values are due to different estimation techniques from the respective representation of $S$-parameters.

Figure $3 \mathrm{~b}$ presents the uncertainty values of 0.01 to $0.75 \mathrm{~dB}$, which are obtained using equations (14) and (15) from the complex representations. The small variations less than $0.03 \mathrm{~dB}$ are found in mismatch uncertainty values, which is due to the difference in the associated uncertainties in $S$-parameters and reflection coefficients. Due to these associated uncertainties, the exact conversion is not accomplished between the magnitude/phase components and the corresponding real/imaginary components. Thus, the responses obtained from the complex values have shown that representations have insignificant effect on the uncertainty evaluation in case of the step attenuator. 


\section{Selection of an attenuator to control the mismatch uncertainty}

In any measurement system, selecting quality components and accessories can minimize the mismatch loss in a welldesigned system, which also increase the overall cost of the system. However, with the applications of few techniques, this uncertainty component can be reduced and restricted to a minimum possible level [12-14]. The most effective technique for minimizing the mismatch uncertainty is to connect the attenuator pads usually of $3 \mathrm{~dB}$, $6 \mathrm{~dB}, 10 \mathrm{~dB}$ at either one port (for power measurement) and both source and load ports (for attenuation measurement) during the measurement. Simultaneously, this also reduces the measurement dynamic range of power and attenuation. The application of attenuators improves the overall mismatch uncertainty with the expense of more complicated mismatch uncertainty formula. For the selection of best attenuator, the attenuator's VSWR should be less than that of the source and load ports.

For a reciprocal attenuator, which has the same VSWR and attenuation in either direction, the actual attenuation level can be expressed in terms of incident and transmitted voltages ( $V_{\text {incident }}$ and $V_{\text {transmitted }}$ ) by the following equation [9],

$$
\begin{aligned}
\text { Attenuation } & =\frac{V_{\text {transmitted }}}{V_{\text {incident }}} \\
& =\frac{10^{-A / 20}}{\left|1 \pm\left(\Gamma_{\mathrm{G}} \Gamma_{\mathrm{A}}+\Gamma_{\mathrm{L}} \Gamma_{\mathrm{A}}+10^{-2 A / 20} \times \Gamma_{\mathrm{G}} \Gamma_{\mathrm{A}}\right)\right|}
\end{aligned}
$$

where, $A$ is the measured attenuation $(\mathrm{dB}),\left|\Gamma_{\mathrm{G}}\right|$ is the reflection coefficient magnitude of source (generator), $\left|\Gamma_{\mathrm{L}}\right|$ is the reflection coefficient magnitude of load (power sensor along with meter or analyzer) and $\left|\Gamma_{\mathrm{A}}\right|$ is the reflection coefficient magnitude of attenuator at both ports.

The voltage uncertainty of equation (16) is given by:

Mismatch uncertanity in voltage

$$
=1 \pm\left(\Gamma_{\mathrm{G}} \Gamma_{\mathrm{A}}+\Gamma_{\mathrm{L}} \Gamma_{\mathrm{A}}+10^{-2 A / 20} \times \Gamma_{\mathrm{G}} \Gamma_{\mathrm{A}}\right) .
$$

Now, if we reconsider the source $V S W R$ is 2.2 and the load $V S W R$ is 1.8 at $2 \mathrm{GHz}$, and vary the attenuator's VSWR value for different attenuation values $3,6,10$ and $40 \mathrm{~dB}$, we can get an impact of attenuation value and $V S W R$ on the mismatch uncertainty. Figure 4 shows that a $40 \mathrm{~dB}$ attenuator offers the least mismatch uncertainty for the same VSWR, by reducing more amount of reflected voltage or power. Thus, a use of higher attenuation reduces the mismatch uncertainty significantly; however, this uncertainty increases with attenuator's VSWR particularly for having $V S W R$ compared to the source and load $V S W R$ values. Higher $V S W R$ of attenuator reflects further voltage or power from the ports towards the generator. When the attenuator value is reduced to 6 or $3 \mathrm{~dB}$, the uncertainty in voltage also changes in a similar manner.

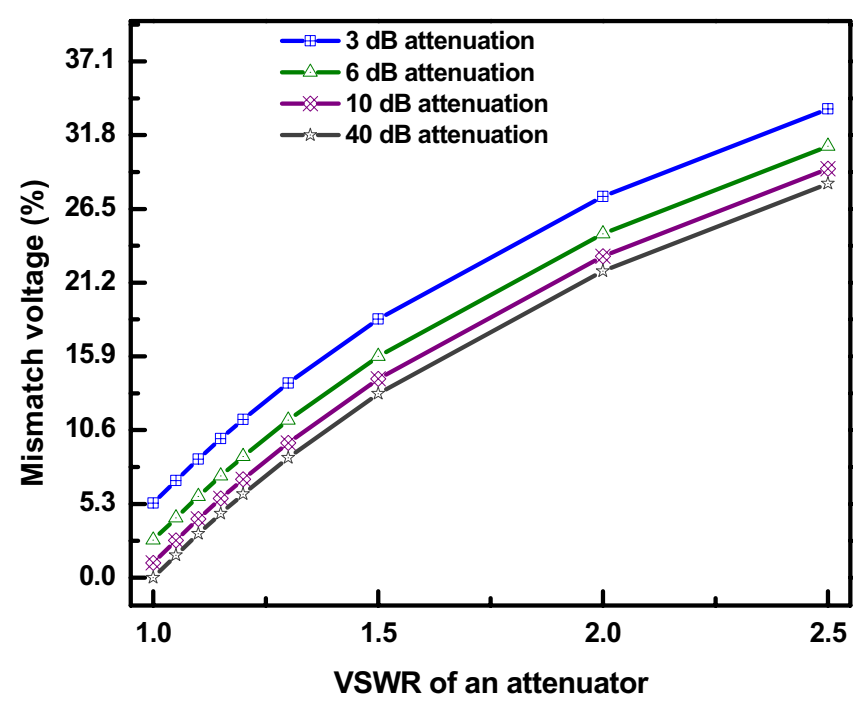

Fig. 4. Effect of attenuator's VSWR on mismatch uncertainty.

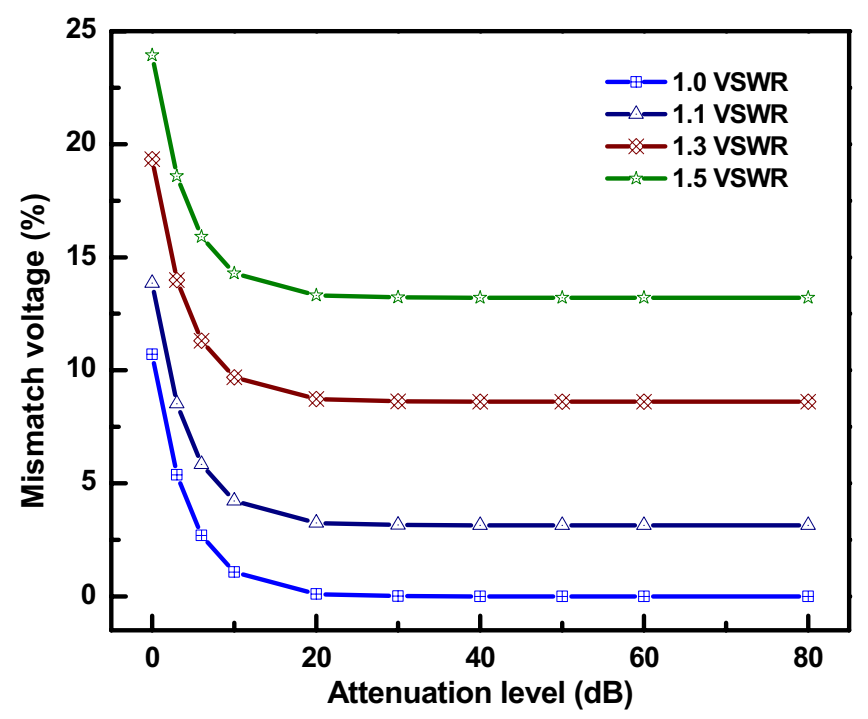

Fig. 5. Effect of attenuation level on mismatch uncertainty.

Now, by varying the attenuation values for different $V S W R$ values of an attenuator, Figure 5 is obtained using equation (17). It shows that to reduce the mismatch uncertainty, increasing the attenuation level is always not the best solution, because the mismatch is dominated by the reflection coefficients of attenuator rather than its attenuation value. So, we agreed that an attenuator of maximum $10 \mathrm{~dB}$ with low $V S W R$ values $(<1.02)$ is optimum for the purpose with compromising the measurement dynamic range. Thus, the attenuation value and its VSWR should be considered in prior to reduce a certain amount of mismatch uncertainty for a given application.

\section{Conclusions}

In this paper, the measurement results of the mismatch uncertainty are presented, which are based on different 
formulas in linear and complex representations for power and attenuation measurement systems. This work verified that the mismatch uncertainty is considerably different, when evaluated from the complex and linear representations for the same DUT in same measurement set-up. Also the mismatch uncertainties are never found same, when these are obtained from the complex representations, whether in magnitude/phase or real/imaginary format. However, these values have shown similar trends in the measurement frequency range, like the uncertainties calculated from reflection coefficient and VSWR values. So, based on this study, we predicted that this variation is due to two factors, first the difference in the individual uncertainty associated with these measured numbers and second, two related quantities may not be converted linearly due to differences in the respective measured values in the operating frequency range. The differences achieved are small but significant as a part of the mismatch uncertainty, which is a dominating contributor in the combined uncertainty of a measured parameter. Thus, the estimation of this uncertainty is found more accurate from the same input representations as of the measured quantity in RF and microwave measurements. For the measurement of large signal, placing a precision attenuator in the measurement path minimizes the mismatch uncertainty preferably of $10 \mathrm{~dB}$. To reduce the measurement uncertainty effectively, the attenuator should be pre-calibrated and should have a very stable characteristic.

Acknowledgements. The author gracefully acknowledges Alan Coster and James Miall, NPL UK in helping the technical contribution of the manuscript. The author is thankful to P.S. Negi, Chief Scientist NPL India for the facilities provided. The present work was performed under NWP-45 project funded by CSIR, India.

\section{References}

1. R.W. Beatty, Mismatch errors in the measurement of ultrahigh frequency and microwave variable attenuators, J. Res. Natl. Bur. Stand. 52, 7-9 (1954)

2. G.E. Schafer, A.Y. Rumfelt, Mismatch errors in the cascade-connected variable attenuators, IRE Trans. Microwave Theory Techn. 7, 447-453 (1959)

3. Microwave mismatch error analysis, Hewlett-Packard Application note 56 (1967)

4. R.W. Beatty, Microwave Attenuation measurements and standards, NBS Monograph 97 (1967)

5. B.D. Hall, Using simulation to check uncertainty calculations, Meas. Sci. Technol. 22, 025105 (2011)

6. B.D. Hall, On the expression of measurement uncertainty for complex quantities with unknown phase, Metrologia 48, 324-332 (2011)

7. K. Patel, P.S. Negi, Importance and estimation of mismatch uncertainty for RF parameters in Calibration laboratories, Int. J. Metrol. Qual. Eng. 3, 29-37 (2012)

8. ISO, ISO/IEC 17025: General requirements for the competence of testing and calibration laboratories (2005)

9. Guidelines on the evaluation and expression of the measurement uncertainty, Singapore Institute of Standards and Industrial Research (1995)

10. Guide to the expression of uncertainty in measurement, Geneva, International Organization for Standardization (1993)

11. Operation Manual, Vector Network Analyzer Model 37247-B, Rev. B, C-12, Wiltron Co., USA (1996)

12. Microwave measurements, edited by R. Collier, D. Skinner (IET Publication, 2009)

13. Coaxial connectors in Radio frequency and microwave measurements, NAMAS, NIS 4303 (1991), Vol. 1

14. D. Skinner, ANAMET Connector guide-Guidance on using Coaxial connectors in measurement, ANAMET report 032 (2001) 